\title{
Patients recevant un médicament du Programme d'accès spécial au sein d'un centre hospitalier universitaire mère-enfant : démarche de surveillance systématique des demandes, de l'utilisation et de l'innocuité des médicaments sur deux années consécutives
}

\author{
Jennifer Corny, Aurélie Guérin, Elaine Pelletier, Suzanne Atkinson, Denis Lebel, Pierre Gaudreault \\ et Jean-François Bussières
}

\section{INTRODUCTION}

L

orsqu'un médicament n'est pas commercialisé dans un pays, Lparce qu'il n'a pas obtenu d'avis de conformité ou d'autorisation (unlicensed), il est parfois possible de l'importer. Au Canada, le "Programme d'accès spécial (PAS) permet aux médecins qui traitent des patients atteints de maladies graves ou mortelles d'accéder à des médicaments non disponibles sur le marché, lorsque les thérapies habituelles se sont révélées inefficaces, ne conviennent pas ou ne sont pas disponibles $»^{1}$. Santé Canada a publié des lignes directrices rappelant qu'il revient au praticien de présenter la demande et de s'assurer que l'utilisation du médicament repose sur des données probantes ${ }^{2}$. Les lignes directrices précisent aussi que le «praticien a la responsabilité de fournir au fabricant de la drogue et au Directeur général un rapport sur les résultats de l'utilisation du médicament dans le cadre du traitement d'urgence, y compris l'information sur les effets indésirables, ainsi que de rendre compte, sur demande, de la quantité de médicament reçue $»^{2}$. L'obtention des médicaments du PAS repose notamment sur le remplissage des formulaires A (Demande d'utilisation précise pour usage immédiat ou à court terme) et B (Demande d'utilisation ultérieure en prévision d'une urgence médicale). Bien que le formulaire $\mathrm{C}$ ait été initialement prévu comme outil de surveillance de l'innocuité par les praticiens, Santé Canada ne l'a pas implanté et l'a finalement retiré des lignes directrices pour confier aux praticiens et aux établissements de santé le soin d'assurer ce suivi ${ }^{3}$. Cependant, ce formulaire reste accessible en ligne sur le portail de Santé-Canada ${ }^{4}$.

Il existe très peu de littérature portant sur l'utilisation de médicaments obtenus par le truchement du PAS au Canada 5 . Dans une analyse réalisée au sein des cinq centres hospitaliers universitaires (CHU) québécois en 2008-2009, le Programme de gestion thérapeutique des médicaments (PGTM) a mis en évidence « la diversité, la répartition et l'importance des [médicaments du] PAS utilisés par les CHU. Pour l'ensemble des CHU, les [médicaments du] PAS ont été l'objet de plus de 12000 ordonnances émises durant l'année analysée [en 2008-2009], ils ont représenté $7,5 \%$ du budget annuel global des médicaments et ont été désignés par plus de 140 dénominations communes $~^{6}$. Comme suite à ces observations, le PGTM a recommandé «que les CHU envisagent d'améliorer et d'uniformiser l'encadrement de l'utilisation des [médicaments du] PAS. Une politique claire, ainsi que des procédures précises et uniformes seraient souhaitables pour améliorer [le recueil et l'archivage de l'information], la surveillance clinique et le financement ainsi que pour améliorer la conformité aux propositions de gestion de Santé Canada $"^{6}$. En réponse à ces préoccupations, nous avons mis en place un nouveau mode de surveillance systématique de l'utilisation et de l'innocuité des médicaments obtenus par le PAS dans notre centre. 


\section{DESCRIPTION DU PROGRAMME}

Il s'agit d'une étude descriptive rétrospective. L'objectif principal était de décrire la démarche de surveillance de l'utilisation et de l'innocuité des médicaments du PAS utilisée pour notre patientèle. L'objectif secondaire visait à présenter un profil des demandes de médicament du PAS et des effets indésirables recensés au cours des deux derniers exercices financiers (c.-̀̀-d. 2012-2013 et 2013-2014).

\section{Lieu et population à l'étude}

Au sein de notre centre hospitalier universitaire mère-enfant de 500 lits, toutes les demandes faites au PAS de Santé Canada par un praticien sont traitées par l'équipe du département de pharmacie. Après la délivrance de l'autorisation par le chef de département de pharmacie, les formulaires A ou B remplis par le praticien sont transmis à Santé Canada. En cas d'autorisation d'importation par l'autorité réglementaire, le département de pharmacie place une commande auprès du fabricant et assure la réception du produit, le suivi et les soins pharmaceutiques requis.

\section{Démarche de surveillance clinique des patients}

Les ordonnances de médicaments obtenues par le PAS pour la période du $1^{\text {er }}$ avril 2012 au 31 mars 2013 et pour la période du $1^{\text {er }}$ avril 2013 au 31 mars 2014 ont été extraites du dossier pharmacologique informatisé contenant le formulaire du statut canadien de chaque médicament rempli manuellement (GesPharx CGSI TI, Québec [Québec]). Les noms des praticiens ayant soumis des demandes de PAS (formulaire A ou B) à Santé Canada et les noms des praticiens ayant prescrit le médicament ont été collectés. Un rapport de surveillance personnalisé établi annuellement a été développé pour chaque praticien et expédié par courrier interne. Si les praticiens n'y répondaient pas, il recevaient un rappel.

Ce rapport était constitué d'une lettre circulaire du chef du département de pharmacie et du président du comité de pharmacologie rappelant au praticien demandeur les modalités d'importation des médicaments accessibles par le PAS et les responsabilités y étant associées ainsi que les modalités de déclaration annuelle d'utilisation et de suivi des effets indésirables liés à l'utilisation de ces médicaments. Annexée à la lettre circulaire se trouve la feuille de suivi (figure 1) regroupant l'ensemble des ordonnances de médicaments applicables aux patients pour qui le praticien avait fait une demande de PAS au cours de l'année. Cette lettre lui permettait de confirmer pour chaque patient la pertinence de la poursuite du traitement et de mentionner la présence éventuelle d'effets indésirables.

\section{Profil des demandes faites au Programme d'accès spécial}

Les données extraites du dossier pharmacologique informatisé (GesPharx CGSI TI) ont été saisies dans un chiffrier (Excel 2010, Microsoft, Seattle [Washington]) en vue d'être analysées. Nous avons calculé le nombre de demandes d'accès à un certain médicament du PAS (une demande pouvait cibler un ou plusieurs patients) et le nombre de transactions informatiques associées à chaque demande. Une transaction informatique correspond à une action inscrite dans le dossier pharmacologique du patient (saisie d'ordonnance ou service de médicament correspondant à une journée de traitement si le patient est hospitalisé ou à une durée variable pour un patient ambulatoire). Le nombre de dénominations communes impliquées, le nombre et les caractéristiques des effets indésirables observés par les

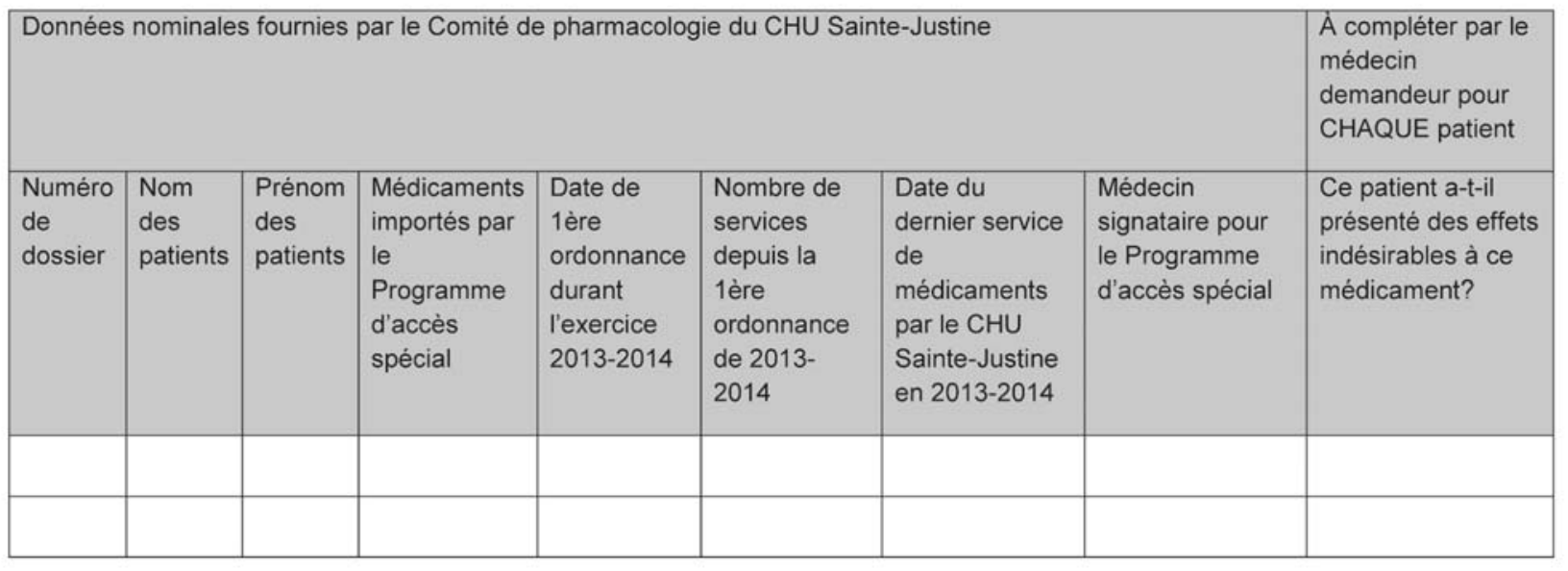

Figure 1. Feuille de suivi médical annuel des patients recevant des médicaments, hospitalisés ou ambulatoires, importés en vertu du Programme d'accès spécial de Santé Canada pour l'exercice financier 2013-2014. 
praticiens ont également été extraits. Nous avons sélectionné le nombre de transactions ayant été effectuées au cours de la période choisie, peu importe la date de début de l'ordonnance. Les préparations magistrales effectuées à partir de produits accessibles par le PAS ont été également comptabilisées.

\section{ÉVALUATION DU PROGRAMME}

\section{Démarche de surveillance clinique des patients}

Pour l'année 2012-2013, l'envoi de la lettre et du tableau personnalisé a été effectué le 8 août 2013 aux 75 praticiens ayant participé au PAS. Deux rappels, respectivement à 34 , puis à deux praticiens ont été envoyés le 18 et le 29 octobre 2013. Trente-huit praticiens ont répondu par écrit au comité de pharmacologie, ce qui correspond à un taux de réponse de $51 \%$ $(n=38 / 75)$ en date du 21 mars 2014.

Pour l'année 2013-2014, l'envoi de la lettre et du tableau personnalisé a été effectué le 22 juillet 2014 à 46 praticiens. Un rappel a été fait le 2 octobre 2014 à 18 praticiens. Trente-deux praticiens ont répondu par écrit au comité de pharmacologie, ce qui donne un taux de réponse de $74 \%(n=34 / 46)$ en date du 27 janvier 2015.

\section{Profil des demandes faites au Programme d'accès spécial}

Pour la période 2012-2013, nous avons recensé 302 demandes de médicaments importés dans le cadre du PAS. Ces demandes concernaient 65 dénominations communes (tableau 1). Pour la période 2013-2014, nous avons recensé 250 demandes. Elles concernaient 65 dénominations communes (tableau 1).

Durant les deux périodes de l'étude, nous avons recensé cinq effets indésirables (quatre pendant la période 2012-2013 et un en 2013-2014) soit un élargissement de l'intervalle QRS (propafénone), une tachycardie à intervalle QRS large (propafénone), une hépatite (névirapine), une hypocalcémie transitoire (ergocalciférol) et une lésion du siège (sévélamer). Tous ces effets indésirables, à l'exception de l'hypocalcémie transitoire sous ergocalciférol, ont conduit à l'arrêt du traitement. Ils correspondaient tous au profil des effets indésirables décrits dans la monographie des médicaments impliqués.

\section{RÉPERCUSSIONS SUR LA PRATIQUE}

À notre connaissance, aucune autre démarche d'encadrement de la surveillance de l'utilisation et de l'innocuité des médicaments accessibles par le PAS n'a été mise en place.

Cette démarche consiste en l'envoi annuel d'une lettre aux praticiens, leur permettant de confirmer la pertinence de la poursuite du traitement des patients ayant reçu un médicament accessible par le PAS et de déclarer la présence d'effets indésirables. Passant de $51 \%$ en 2012-2013 à $74 \%$ en 2013-2014, le taux de réponse des praticiens a considérablement augmenté pendant la durée de notre étude. Cette augmentation peut s'expliquer par une meilleure compréhension du processus au cours de la deuxième année du Programme, mais également par une prise de conscience des praticiens de la nécessité de justifier la pertinence du traitement et de rapporter l'innocuité des médicaments importés en leur nom au Canada. L'envoi systématique de cette lettre permet également une communication entre les pharmaciens et les praticiens afin de faciliter la déclaration d'effets indésirables.

Notre analyse a permis de décrire le profil de ces demandes dans notre CHU. Entre les deux années, on a noté que les produits utilisés dans le cadre du PAS étaient différents. Outre les besoins cliniques des patients qui varient dans le temps, plusieurs raisons peuvent expliquer ces différences. Tout d'abord, certains médicaments ont obtenu un avis de conformité de Santé Canada au cours de ces deux périodes (Diacomit ${ }^{\text {md }}$ [stiripentol] en 2013). Puis est intervenue l'obtention d'autorisations d'importation de nouveaux médicaments par le PAS (p. ex. granulocyte-macrophage colony-stimulating factor [facteur de stimulation des colonies de granulocytes et de macrophages]). Dans certains cas, le fabricant a refusé de vendre un produit au Canada ou un médicament a été retiré de la liste du PAS (p. ex. la propafénone dosée à $10 \mathrm{mg}$, provenant d'un fabricant allemand). De plus, de nouvelles options ont été mises à disposition sur le marché canadien (p. ex. Intuniv $\mathrm{XR}^{\mathrm{md}}$ [guanfacine]). Enfin, les pénuries de médicaments ont entrainé le recours à des médicaments accessibles par le PAS (p. ex. le triméthoprimsulfaméthoxazole en solution orale provenant de $\mathrm{Hi}$-Tech Pharma durant la pénurie du fabricant Teva).

Si les praticiens ont confirmé la pertinence de poursuivre les traitements dans tous les cas de suivi, il faut noter un taux de réponses finales des praticiens à la circulaire accru $(51 \%$ c. $74 \%)$ et un nombre plus limité de praticiens interpellés (de 75 à 46). Cette réduction du nombre de praticiens nous semble liée à la mise en place du Programme local d'encadrement qui responsabilise davantage les personnes concernées et qui met en évidence les obligations d'exercer un suivi.

Peu d'effets indésirables ont été rapportés au cours des deux exercices. Ils concernaient cinq demandes sur 552 demandes prises en compte, soit $0,9 \%$. Ces effets indésirables faisaient partie de ceux connus et rapportés dans les monographies en vigueur au sein des pays ayant commercialisé ces produits.

Cette étude descriptive confirme la pertinence de la mise en place par les CHU d'un programme structuré de surveillance clinique des médicaments obtenus par le PAS.

Cette étude et cette démarche comportent toutefois des limites. Étant donné le caractère rétrospectif de l'étude, nous ne savons pas si les praticiens ont rapporté les effets indésirables à Santé Canada ou au fabricant. De plus, les transactions informatiques sont comptabilisées dans l'exercice financier en vigueur, 
Tableau 1. Profil des demandes et des transactions informatiques du Programme d'accès spécial (PAS) pour les exercices 2012-2013 et 2013-2014*

2012-2013

2013-2014

\begin{tabular}{|c|c|c|c|c|}
\hline Produit pharmaceutique & $\begin{array}{l}\text { Nombre de } \\
\text { demandes }\end{array}$ & $\begin{array}{c}\text { Nombre de } \\
\text { transactions } \\
\text { informatiques }\end{array}$ & $\begin{array}{l}\text { Nombre de } \\
\text { demandes }\end{array}$ & $\begin{array}{c}\text { Nombre de } \\
\text { transactions } \\
\text { informatiques }\end{array}$ \\
\hline acétaZOLAMIDE 250 mg par capsule & 1 & 15 & 1 & 12 \\
\hline acide carglumique 200 mg en comprimés & 1 & 2 & 1 & 0 \\
\hline $\begin{array}{l}\text { acide dimercaptosuccinique (DMSA) } \\
100 \text { mg en capsules }\end{array}$ & 1 & 4 & 0 & 0 \\
\hline acide valproique $100 \mathrm{mg} / \mathrm{mL}$ fiole injectable & 1 & 35 & 2 & 1 \\
\hline acyclovir $3 \%$ tube onguent ophtalmique & 0 & 0 & 0 & 2 \\
\hline albendazole 200 mg en comprimés & 1 & 9 & 1 & 0 \\
\hline artesunate $110 \mathrm{mg}$ fiole injectable & 0 & 0 & 0 & 7 \\
\hline L-asparaginase pegylée $750 \mathrm{Ul} / \mathrm{mL}$ injectable & 23 & 119 & 11 & 47 \\
\hline aztréonam $1 \mathrm{~g}$ fiole injectable & 1 & 14 & 0 & 0 \\
\hline bumétanide $0,5 \mathrm{mg} / \mathrm{mL}$ ampoule injectable & 0 & 0 & 1 & 18 \\
\hline cafeine citrate $1 \mathrm{mg} / \mathrm{mL}$ injectable & 3 & 2029 & 4 & 2166 \\
\hline carboxypeptidase $1000 \mathrm{UI}$ & 1 & 1 & 0 & 0 \\
\hline ch14.18 sac injectable & 0 & 0 & 12 & 3 \\
\hline chlorothiazide $500 \mathrm{mg}$ fiole injectable & 0 & 6 & 2 & 14 \\
\hline cidofovir 375 mg injectable & 5 & 163 & 5 & 258 \\
\hline $\begin{array}{l}\text { cisapride } 10 \mathrm{mg} \text { en comprimés et } \\
1 \mathrm{mg} / \mathrm{mL} \text { seringue solution orale }\end{array}$ & 41 & 556 & 25 & 1168 \\
\hline cysteamine en capsules $50 \mathrm{mg} / 150 \mathrm{mg}$ & 6 & 226 & 7 & 131 \\
\hline défibrotide sac injectable & 4 & 87 & 7 & 148 \\
\hline deflazacort 6 mg en comprimés & 6 & 28 & 7 & 20 \\
\hline disopyramide $100 \mathrm{mg}$ en capsules & 2 & 0 & 0 & 0 \\
\hline divalproex sodique $125 \mathrm{mg}$ en capsules & 12 & 161 & 9 & 149 \\
\hline doxycycline $100 \mathrm{mg}$ fiole injectable & 3 & 30 & 3 & 35 \\
\hline $\begin{array}{l}\text { echothiophate (iodure) 0,125 \% bouteille } \\
\text { gouttes ophtalmiques }\end{array}$ & 2 & 37 & 3 & 31 \\
\hline efavirenz $30 \mathrm{mg} / \mathrm{mL}$ solution orale & 0 & 8 & 0 & 0 \\
\hline émulsion lipidique de poisson $10 \%$ & 12 & $\mathrm{AP}$ & 20 & $\mathrm{AP}$ \\
\hline $\begin{array}{l}\text { ergocalciferol (vi.D2) } 400000 \mathrm{Ul} / \mathrm{mL} \\
\text { ampoule injectable }\end{array}$ & 2 & 16 & 3 & 9 \\
\hline esmolol $10 \mathrm{mg} / \mathrm{mL}$ fiole injectable & 0 & 0 & 2 & 0 \\
\hline etomidate $2 \mathrm{mg} / \mathrm{mL}$ fiole injectable & 2 & 0 & 2 & 0 \\
\hline felbamate 600 mg en comprimés & 18 & 168 & 18 & 237 \\
\hline flucytosine $500 \mathrm{mg}$ en capsules & 1 & 0 & 1 & 0 \\
\hline foscarnet $24 \mathrm{mg} / \mathrm{mL}$ injectable & 5 & 146 & 5 & 147 \\
\hline galsulfase $1 \mathrm{mg} / \mathrm{mL}$ injectable & 5 & 79 & 2 & 41 \\
\hline gemtuzumab sac injectable & 1 & 9 & 0 & 0 \\
\hline GM-csf $250 \mu \mathrm{g} / \mathrm{mL}$ seringue injectable & 0 & 0 & 2 & 30 \\
\hline GM-csf $500 \mu \mathrm{g} / \mathrm{mL}$ seringue injectable & 2 & 0 & 0 & 2 \\
\hline guanfacine 1 mg en comprimés & 11 & 69 & 7 & 57 \\
\hline hyaluronidase $1500 \mathrm{UI}$ ampoule injectable & 1 & 19 & 2 & 15 \\
\hline $\begin{array}{l}\text { hydrocortisone (cypionate d') } 2 \mathrm{mg} / \mathrm{mL} \\
\text { solution orale }\end{array}$ & 0 & 1 & 0 & 1 \\
\hline $\begin{array}{l}\text { hydroxocobalamine } 500 \mu \mathrm{g} / \mathrm{mL} \\
\text { fiole injectable }\end{array}$ & 1 & 244 & 1 & 251 \\
\hline ibuprofène $5 \mathrm{mg} / \mathrm{mL}$ ampoule injectable & 4 & 340 & 10 & 449 \\
\hline iloprost $10 \mu \mathrm{g} / \mathrm{mL}$ ampoule solution nébulisation & 3 & 4 & 0 & 4 \\
\hline
\end{tabular}


Tableau 1. Profil des demandes et des transactions informatiques du Programme d'accès spécial (PAS) pour les exercices 2012-2013 et 2013-2014* (suite)

2012-2013

2013-2014

\begin{tabular}{|c|c|c|c|c|}
\hline Produit pharmaceutique & $\begin{array}{l}\text { Nombre de } \\
\text { demandes }\end{array}$ & $\begin{array}{c}\text { Nombre de } \\
\text { transactions } \\
\text { informatiques }\end{array}$ & $\begin{array}{l}\text { Nombre de } \\
\text { demandes }\end{array}$ & $\begin{array}{c}\text { Nombre de } \\
\text { transactions } \\
\text { informatiques }\end{array}$ \\
\hline indomethacine $1 \mathrm{mg}$ injectable & 0 & 0 & 3 & 0 \\
\hline interferon gamma injectable & 0 & 0 & 1 & 3 \\
\hline ivermectin 3 mg en comprimés & 4 & 10 & 0 & 0 \\
\hline levetiracetam 100 mg/mL solution orale & 1 & 0 & 0 & 0 \\
\hline $\begin{array}{l}\text { levosimendan } 2,5 \mathrm{mg} / \mathrm{mL} \text { fiole injectable } \\
\text { et } 0,05 \mathrm{mg} / \mathrm{mL} \text { sac injectable }\end{array}$ & 9 & 118 & 0 & 0 \\
\hline liothyronine (T3) $10 \mu \mathrm{g} / \mathrm{mL}$ fiole injectable & 2 & 69 & 3 & 64 \\
\hline mafenide $8,5 \%$ tube crème & 3 & 16 & 2 & 8 \\
\hline mercaptamine 75 mg en capsules & 0 & 0 & 1 & 0 \\
\hline méthadone $10 \mathrm{mg} / \mathrm{mL}$ ampoule injectable & 1 & 0 & 3 & 4 \\
\hline mifepristone 200 mg en comprimés & 4 & 28 & 4 & 39 \\
\hline mupirocine $2 \%$ tube onguent nasal & 0 & 7 & 0 & 15 \\
\hline nevirapine $10 \mathrm{mg} / \mathrm{mL}$ solution orale & 10 & 32 & 5 & 76 \\
\hline $\begin{array}{l}\text { niCARdipine } 0,1 \mathrm{mg} / \mathrm{mL} \text { et } 0,2 \mathrm{mg} / \mathrm{mL} \\
\text { sac injectable }\end{array}$ & 2 & 26 & 1 & 118 \\
\hline niCARdipine 2,5 mg/mL ampoule injectable & 2 & 60 & 0 & 0 \\
\hline $\begin{array}{l}\text { NTBC [2-(2-nitro-4-trifluorométhyl- } \\
\text { benzoyl)-1,3-cyclohexanedione; } \\
\text { nitisinone] en capsules } 2 \text { mg/5 mg/10 mg }\end{array}$ & 4 & 736 & 4 & 767 \\
\hline pegademase $250 \mathrm{Ul} / \mathrm{mL}$ seringue injectable & 0 & 0 & 1 & 31 \\
\hline $\begin{array}{l}\text { PENTobarbital } 50 \mathrm{mg} / \mathrm{mL} \text { fiole injectable } \\
\text { et sac injectable }\end{array}$ & 2 & 33 & 7 & 32 \\
\hline physostigmine $1 \mathrm{mg} / \mathrm{mL}$ injectable & 1 & 0 & 0 & 0 \\
\hline $\begin{array}{l}\text { phytonadione (vitamine K1) } 5 \text { mg } \\
\text { en comprimés }\end{array}$ & 3 & 223 & 2 & 35 \\
\hline piracetam 1200 mg en comprimés & 0 & 0 & 2 & 28 \\
\hline ponatinib 15 mg en comprimés & 2 & 25 & 1 & 4 \\
\hline potassium phosphate 305 mg en comprimés & 1 & $\mathrm{AP}$ & 1 & AP \\
\hline propafenone 10 mg en comprimés & 5 & 70 & 3 & 37 \\
\hline protireline $200 \mu \mathrm{g} / \mathrm{mL}$ en ampoule injectable & 3 & 4 & 2 & 2 \\
\hline ribavirine 100 mg/ml injectable & 2 & 14 & 2 & 84 \\
\hline $\begin{array}{l}\text { rifampicine } 600 \mathrm{mg} \text { fiole injectable et } \\
60 \mathrm{mg} / \mathrm{mL} \text { fiole injectable }\end{array}$ & 11 & 166 & 5 & 63 \\
\hline rifaximine 550 mg en comprimés & 2 & 0 & 0 & 0 \\
\hline sacrosidase $8500 \mathrm{Ul} / \mathrm{mL}$ solution orale & 1 & 1 & 3 & 9 \\
\hline sevelamer (carbonate) 2,4 g sachet & 0 & 0 & 1 & 4 \\
\hline $\begin{array}{l}\text { sodium glycerophosphonate } \\
1 \mathrm{mmol} / \mathrm{mL} \text { injectable }\end{array}$ & 2 & AP & 2 & AP \\
\hline $\begin{array}{l}\text { sodium phenylacetate/sodium benzoate } \\
10 \mathrm{mg} / \mathrm{mL} \text { sac injectable }\end{array}$ & 1 & 12 & 2 & 17 \\
\hline $\begin{array}{l}\text { sodium (phenylbutyrate) } 500 \mathrm{mg} \\
\text { comprimé et } 200 \mathrm{mg} / \mathrm{mL} \text { solution orale }\end{array}$ & 1 & 124 & 3 & 21 \\
\hline stiripentol 250 mg en capsules & 20 & 203 & NA & NA \\
\hline sulfaDIAZine 500 mg en comprimés & 2 & 7 & 0 & 0 \\
\hline sulthiame 50 mg en comprimés & 1 & 50 & 2 & 26 \\
\hline thiotepa $100 \mathrm{mg}$ et $15 \mathrm{mg}$ injectable & 5 & 0 & 3 & 0 \\
\hline tinidazole 500 mg en comprimés & 1 & 0 & 0 & 0 \\
\hline tocopherol alfa injectable & 1 & $\mathrm{AP}$ & 1 & AP \\
\hline
\end{tabular}


This single copy is for your personal, non-commercial use only.

For permission to reprint multiple copies or to order presentation-ready copies for distribution, contact CJHP at cjhpedit@cshp.ca

\section{Tableau 1. Profil des demandes et des transactions informatiques du Programme d'accès spécial (PAS) pour les exercices 2012-2013 et 2013-2014* (suite et fin)}

2012-2013

2013-2014

\begin{tabular}{lcccc}
\cline { 2 - 5 } Produit pharmaceutique & $\begin{array}{c}\text { Nombre de } \\
\text { demandes }\end{array}$ & $\begin{array}{c}\text { Nombre de } \\
\text { transactions } \\
\text { informatiques }\end{array}$ & $\begin{array}{c}\text { Nombre de } \\
\text { demandes }\end{array}$ & $\begin{array}{c}\text { Nombre de } \\
\text { transactions } \\
\text { informatiques }\end{array}$ \\
\hline $\begin{array}{l}\text { triamcinolone (hexacetonide) } 20 \mathrm{mg} / \mathrm{mL} \\
\text { ampoule injectable }\end{array}$ & 6 & 1 & 4 & 0 \\
\hline trientine $250 \mathrm{mg}$ en capsules & 4 & 39 & 4 & 26 \\
\hline $\begin{array}{l}\text { trimethoprim } 8 \mathrm{mg} / \mathrm{mL}+\text { sulfamethoxazole } \\
40 \mathrm{mg} / \mathrm{mL} \text { solution orale }\end{array}$ & 1 & 149 & 2 & 0 \\
\hline vitamines liposolubles & 0 & $\mathrm{AP}$ & 2 & $\mathrm{AP}$ \\
\hline vitamines hydrosolubles & 0 & $\mathrm{AP}$ & 1 & $\mathrm{AP}$ \\
\hline zanamivir $200 \mathrm{mg} / 20 \mathrm{~mL}$ solution orale & 0 & 0 & 0 & 0 \\
\hline zonisamide $100 \mathrm{mg}$ en comprimés & 0 & 3 & 250 & 0 \\
\hline Total & 302 & 6851 & 986 \\
\hline AP
\end{tabular}

$\overline{\mathrm{AP}}=$ alimentation parentérale (ces produits étant uniquement utilisés comme ajouts à des préparations destinées à l'alimentation parentérale, aucune transaction informatique n'est comptabilisée dans le logiciel de prescription nominative), GM-csf = granulocytemacrophage colony-stimulating factor (facteur de stimulation des colonies de granulocytes et de macrophages), NA = non applicable (le stiripentol a obtenu un avis de conformité en 2013, et aucune demande dans le cadre du PAS n'a été soumise par la suite). * Une transaction informatique correspond à une action posée par un pharmacien ou un assistant technique en pharmacie inscrite dans le dossier pharmacologique informatisé; une transaction peut inclure une saisie d'ordonnance ou un service. Ceci illustre le volume d'activité relative entre les différents médicaments. Un médicament obtenu par le PAS peut faire l'objet d'une transaction pour une année donnée, même si la demande d'importation a été réalisée lors d'un exercice financier précédent. De même, une demande d'importation peut ne comporter aucune transaction informatique si la dispensation survient lors d'un autre exercice financier.

bien que la demande d'accès au PAS et l'ordonnance aient pu être réalisées au cours de l'année précédente. Plusieurs praticiens n'ont pas été en mesure de remplir le formulaire de demande de suivi dans les cas d'utilisation ultérieure. En effet, dans les cas d'utilisation antérieure, le praticien demandeur n'est pas toujours le praticien qui prescrit effectivement le médicament pour un patient donné. De plus, notre démarche nous permet de retrouver les effets indésirables à discuter au comité de pharmacologie et à déclarer au Programme de Santé Canada. Le système mis en place devrait être amélioré pour tenir compte de façon plus systématique des prescripteurs qui font des demandes destinées à une utilisation ultérieure. Cette démarche a aussi mis en évidence la nécessité d'améliorer le recueil des renseignements sur les médicaments comportant peu de données probantes. Un encadrement encore plus élaboré visant à assurer la surveillance clinique des médicaments considérés comme émergents est actuellement à l'étude et en cours d'implantation dans notre centre ${ }^{7,8}$. Cette démarche permet d'encadrer la prescription, la justification, l'autorisation, l'utilisation et la surveillance de ces médicaments, grâce à une collaboration des pharmaciens et prescripteurs. Grâce à cet encadrement, des cibles thérapeutiques précises sont définies et versées au dossier du patient (paramètres à suivre, valeur cible et délai de réponse) ainsi que des marqueurs d'innocuité (effet indésirable à suivre, fréquence de suivi, modalités de prise en charge en cas d'apparition d'effets indésirables).

\section{CONCLUSION}

Cette étude décrit une démarche locale permettant l'encadrement de l'utilisation et la surveillance de l'innocuité des médicaments obtenus par le PAS au sein d'un établissement universitaire. Cette démarche s'inscrit dans le cadre du contrôle de la qualité des soins fournis à nos patients. Bien que le taux de réponse des prescripteurs ou demandeurs n'était que de $74 \%$ après deux années d'implantation, le Programme a permis de sensibiliser les intervenants et de déterminer cinq effets indésirables associés à ces médicaments. La surveillance de l'utilisation et de l'innocuité des médicaments du PAS revient aux praticiens, et le comité de pharmacologie peut jouer un rôle efficace dans le cadre de ce programme.

\section{Références}

1. Médicaments et produits de santé: médicaments. Ottawa $(\mathrm{ON})$ : Santé Canad; 2015. Publié au : www.hc-sc.gc.ca/dhp-mps/acces/drugs-drogues/indexfra.php. Consulté le 22 juillet 2015.

2. Ligne directrice à l'intention de l'industrie et des praticiens - Programme d'accès spécial - médicaments. Ottawa $(\mathrm{ON})$ : Santé Canada; 2013. Publié au : www.hc-sc.gc.ca/dhp-mps/acces/drugs-drogues/sapg3_pasg3-fra.php\#a1. Consulté le $1^{\text {er juin } 2015 .}$

3. Bussières JF. Législation et systèmes de soins. $10^{\circ}$ édition. Montréal (QC) : Publié à compte d'auteur; 2014. p. 245.

4. Formulaire de suivi Formulaire C. Ottawa (ON) : Santé Canada; 2008. Publié au : www.hc-sc.gc.ca/dhp-mps/acces/drugs-drogues/sapf3_pasf3-fra.php. Consulté le $1^{\text {er juin } 2015 .}$ 
5. Atkinson S, Blanc A, Lebel D, Bussières JF, Bailey B, Bérard A. Risk of drug interactions among children accessing drugs through Health Canada's Special Access Programme. Can J Hosp Pharm 2007;60(2):114-20.

6. Médicaments obtenus par le Programme d'accès spécial de Santé Canada dans les CHU du Québec : 2008-2009. Montréal (QC) : Programme de gestion thérapeutique des médicaments. 2009. Publié au : http://pgtm.org/ documentation/FSW/PAS_AD_Rapport_final_20101208.pdf. Consulté le $1^{\text {er }}$ juin 2015.

7. Corny J, Pelletier E, Lebel D, Bussières JF. Retrospective review of emerging drug use in a mother-child center in Quebec [résumé]. Can J Hosp Pharm. 2015;68(1):82.

8. Corny J, Tanguay C, Pelletier E, Lebel D, Bussières JF. Encadrement de l'émergence des médicaments dans un centre hospitalier universitaire mèreenfant [résumé]. J Popul Ther Clin Pharmacol. 2014;21(2):e320s.

Jennifer Corny est candidate au D. Pharm. et interne en pharmacie, Université Paris Descartes, Paris, France, et assistante de recherche à l'Unité de recherche en pratique pharmaceutique, Centre hospitalier universitaire Sainte-Justine, Montréal (Québec).

Aurélie Guérin est candidate au D. Pharm. et interne en pharmacie, Université Paris Sud XI, France, et assistante de recherche à I'Unité de recherche en pratique pharmaceutique, Centre hospitalier universitaire Sainte-Justine, Montréal (Québec).
Elaine Pelletier, B. Pharm, M. Sc., est pharmacienne, Département de pharmacie, Centre hospitalier universitaire Sainte-Justine, Montréa (Québec).

Suzanne Atkinson, B. Pharm, M. Sc., est Adjointe, Département de pharmacie, Centre hospitalier universitaire Sainte-Justine, Montréal (Québec).

Denis Lebel, B. Pharm, M.Sc., FCSHP, est Adjoint, Département de pharmacie, Centre hospitalier universitaire Sainte-Justine, Montréal (Québec)

Pierre Gaudreault, M. D., est pédiatre, Département de pédiatrie, Centre hospitalier universitaire Sainte-Justine, Montréal (Québec).

Jean-François Bussières, B. Pharm., M. Sc., FCSHP, est Chef, Département de pharmacie et Unité de recherche en pratique pharmaceutique, Centre hospitalier universitaire Sainte-Justine, et professeur adjoint de clinique, Faculté de pharmacie, Université de Montréal, Montréal (Québec)

Intérêts concurrents : Aucun déclaré

\section{Adresse de correspondance :}

Jean-François Bussières

Département de pharmacie et Unité de recherche

en pratique pharmaceutique

Centre hospitalier universitaire Sainte-Justine

3175, chemin de la Côte Sainte-Catherine

Montréal QC

H3T 1C5

Courriel : jf.bussieres@ssss.gouv.qc.ca

\section{Compounding: Guidelines for Pharmacies}

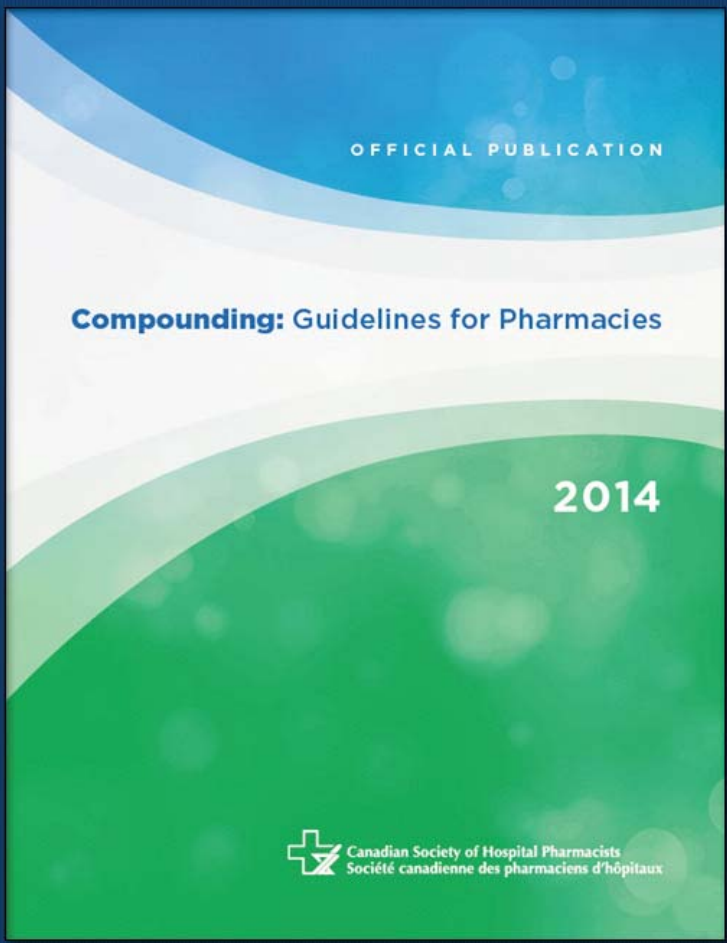

These comprehensive guidelines cover compounding in pharmacies where the compounded preparations are intended for human use, regardless of route of administration or whether the preparation is related to research purposes. The guidelines also apply to the preparation of radiopharmaceuticals and other hazardous pharmaceuticals.

Available in English

(French translation in progress)

Order your copy at www.CSHP.ca 